Palavras chave: Formigas cortadeiras Método de controle Eucaliptos Mecanização florestal

Histórico:

Recebido 03/10/201 I Aceito 15/04/2015

Keywords: Leaf-cutting ants Control method Eucalyptus Forestry mechanization

Correspondence: marcelo.areis@hotmail.com

DOI:
Marcelo de Almeida Reis', João Paulo Arantes Rodrigues Cunha², Ronald Zanetti ${ }^{3}$, Bianca Vique Fernandes ${ }^{4}$, Janaine Myrna Rodrigues Reis ${ }^{5}$

\section{APLICAÇÃO SISTEMÁTICA MECANIZADA DE ISCA FORMICIDA GRANULADA EM EUCALIPTAIS EM FASE DE MANUTENÇÃO}

RESUMO: O controle sistemático mecanizado com isca formicida em eucaliptais demanda menos mão de obra, menor contaminação dos trabalhadores e maior rendimento operacional, quando comparado com o controle manual. O objetivo deste trabalho foi avaliar a mortalidade de ninhos de formigas cortadeiras com aplicação sistemática mecanizada de isca formicida em três espaçamentos em eucaliptais em fase de manutenção, a capacidade operacional da aplicação mecanizada e a qualidade de distribuição de isca ao longo da área. $O$ experimento foi realizado no município de Paraopeba, Minas Gerais, em talhões de eucaliptos em fase de manutenção. Foi aplicado I kg.ha-1 de isca formicida granulada a base de sulfluramida $(0,3 \%)$ sistematicamente a cada $6 \times 2,5 \mathrm{~m}, 9 \times 2,5 \mathrm{~m}$ e $12 \times 2,5 \mathrm{~m}$, com um equipamento de aplicação acoplado ao sistema hidráulico de um trator, constituindo três tratamentos com cinco repetições. Foram selecionados e marcados ninhos com tamanho de até um metro quadrado de área de terra e avaliado sua inatividade aos 60 e 210 dias após aplicação da isca formicida. Também foram avaliadas a percentagem de carregamento da isca, a capacidade operacional da operação mecanizada e a qualidade do processo de distribuição da isca, por meio de cartas de controle. Verificou-se que os diferentes espaçamentos empregados não influenciaram a eficácia de controle. $O$ maior espaçamento empregado proporcionou maior capacidade operacional $\left(8,42 \mathrm{ha} \cdot \mathrm{h}^{-1}\right)$. A aplicação sistemática mecanizada proporcionou boa qualidade de distribuição, sob ponto de vista do controle estatístico de processo, constituindo alternativa viável a aplicação manual.

\section{MECHANIZED SYSTEMATIC APPLICATION OF GRANULETED ANTS BAITS IN EUCALYPTUS PLANTATIONS IN MAINTENANCE PHASE}

ABSTRACT: Compared to manual control, systematic control WITH ant bait requires less labor, results in lower exposure of workers and increases operating YIELD. This study aimed to evaluate the mortality of leaf-cutting ants nests with a mechanized systematic application of ant bait in three spacings on eucalyptus plantations in maintenance phase, the operational capability of mechanized application and the quality of the distribution of bait throughout the area. The experiment was conducted in the city of Paropeba, Minas Gerais, Brazil, in stands of eucalyptus plantations in maintenance phase. A sulfluramid (0.3\%) based bait was systematically applied at I $\mathrm{kg} \cdot \mathrm{ha}^{-1}$ every $6 \times 2.59 \times 2.5$ and $12 \times 2.5$ meters, with an application equipment attached to the hydraulic system of a tractor, comprising three treatments with five repetitions. Nests with a size of up to one square meter of land area were selected and marked and evaluated their inactivity at 60 and 210 days after bait application. The percentage of bait loading, the operational capability of the mechanized process and the quality of the distribution process of the bait were also evaluated, using control charts. It was found that the different spacings applied did not influence the effectiveness of control. The largest spacing used provided greater operational capacity (8.42 ha. $\left.\mathrm{h}^{-1}\right)$. The mechanized systematic application provided good distribution as to the process' statistical control, being a viable alternative to manual application.

\footnotetext{
I IMFLOR- Inventário Manejo e Monitoramento Florestal Ltda, Lavras, Minas Gerais, Brasil

${ }^{2}$ Universidade Federal de Uberlândia, Uberlândia, Minas Gerais, Brasil

${ }^{3}$ Universidade Federal de Lavras, Lavras, Minas Gerais, Brasil

${ }^{4}$ Vallourec Florestal, Paraopeba, Minas Gerais, Brasil

${ }^{5}$ Centro Universitário de Patos de Minas, Patos de Minas, Minas Gerais, Brasil
} 


\section{INTRODUÇÃO}

Formigas cortadeiras dos gêneros Atta e Acromyrmex (Hymenoptera: Formicidae) são os insetos que mais causam problemas para plantios florestais no Brasil (NICHOLS-ORIANS, I99I; ZANETTI et al., 2003). O controle de seus ninhos em eucaliptais é realizado por meio do uso iscas formicidas granuladas de forma localizada no ninho ou sistemática na área, independentemente da localização dos mesmos (ANDRADE et al., 2002; CAMARGO et al., 2003; LOPES et al., 2003; NAGAMOTO et al., 2007).

O controle sistemático com iscas granuladas é comum nas fases iniciais da floresta cultivada, mas sua efetividade no controle operacional dessa praga em fase de manutenção é pouca estudada, principalmente de forma mecanizada. A aplicação sistemática pode reduzir a quantidade de mão-de-obra dessa operação e minimizar a exposição dos trabalhadores à contaminação com inseticidas. Esse tipo de controle de ninhos foi avaliado para Acromyrmex spp. e Atta spp. com isca formicida a granel e micro porta-isca em áreas de reforma de eucalipto com cultivo mínimo por Zanetti et al. (2003). A eficiência de micro porta-isca aplicado sistematicamente para o controle de Acromyrmex spp. também foi avaliada com diferentes dosagens e tempos após o preparo do solo por Ukan (2008). Reis et al. (2009) estudaram a aplicação sistemática mecanizada de isca granulada na dose de I kg.ha-1 na região do Cerrado Mineiro, com eficiência de mortalidade de $87,33 \%$ dos ninhos de Atta sexdens rubropilosa.

A avaliação da melhor forma de aplicação de iscas e sua eficácia são importantes para o controle de formigas cortadeiras. Além disto, permite obter um controle mais efetivo e com menor impacto ambiental (NAGAMOTO et al., 2004, 2007; REIS et al., 2008). A mecanização neste processo ainda é incipiente e pouco se conhece sobre o desempenho operacional dos aplicadores de iscas granuladas tratorizados, incluindo capacidade operacional e qualidade de distribuição. Também ainda não se tem definido o espaçamento ideal de distribuição de isca ao longo das áreas tratadas, o que interfere diretamente no custo da produção e na efetividade de controle.

O controle estatístico do processo (CEP) é um conjunto de técnicas úteis para resolução de problemas, alcance da estabilidade do processo e aumento da capacidade por meio da redução da variabilidade (MONTGOMERY, I99I). O CEP tem como objetivo detectar rapidamente alterações dos parâmetros de determinados processos para que os problemas possam ser corrigidos antes que muitos itens não conformes sejam produzidos (MINGOTI; FIDELIS, 200I).

O controle de qualidade é perfeitamente adaptável ao sistema de produção agrícola; sabe-se que, com a correção e a eliminação de desperdícios e falhas, inúmeras vantagens serão acrescidas à competitividade do campo (BONILLA, I994). De acordo com Montgomery (I99I), a carta de controle é uma das principais ferramentas utilizadas no controle estatístico da qualidade.

Desta forma, o objetivo deste trabalho foi avaliar a mortalidade de ninhos de formigas cortadeiras com aplicação sistemática mecanizada de isca formicida; o rendimento da aplicação mecanizada; a percentagem de carregamento da isca e a qualidade da distribuição de isca ao longo da área tratada, por meio de cartas de controle em três espaçamentos em eucaliptais em fase de manutenção no Cerrado do Estado de Minas Gerais, Brasil.

\section{MATERIAL E MÉTODOS}

O experimento foi realizado em área comercial (V\&M Florestal) no município de Paraopeba, Estado de Minas Gerais, Brasil. Foram utilizados talhões de eucaliptos em fase de manutenção com idade de 3 anos e espaçamento de plantio de $3 \times 2 \mathrm{~m}$, totalizando 76 hectares, com infestação de ninhos de Atta sexdens rubropilosa (Forel, 1908) (Hymenoptera: Formicidae). Foi aplicado I $\mathrm{kg}$ de isca formicida granulada a base de sulfluramida $(0,3 \%)$ por hectare utilizando os espaçamentos de $6 \times 2,5 \mathrm{~m}, 9 \times 2,5 \mathrm{~m}$ e $12 \times 2,5 \mathrm{~m}$, constituindo três tratamentos. A quantidade de isca de $\mathrm{I} \mathrm{kg} \cdot \mathrm{ha}^{-1}$ é a menor dose economicamente viável que pode ser aplicada de forma sistemática mecanizada (REIS et al., 2009).

Empregou-se um aplicador de isca formicida granulada, em que a dose é controlada por um sistema de rosca sem fim acionada eletricamente por um temporizador. A distância entre pontos de distribuição é regulada por um controlador eletrônico e um sensor de ponto instalado na roda traseira do trator. $O$ equipamento, desenvolvido pelas empresas Unibrás e Karmac, foi acoplado ao sistema hidráulico de um trator $(55,2 \mathrm{~kW}$ de potência), e estava dotado de uma linha de distribuição. $O$ equipamento aplica isca de forma sistemática e intermi- 
tente no solo ou sob a serrapilheira existente. $O$ trator foi operado à velocidade média de $6 \mathrm{~km} \cdot \mathrm{h}^{-1}$, com pequenas variações dependendo das condições de topografia e densidade de sub-bosque do talhão.

O delineamento experimental utilizado foi inteiramente casualizado (DIC), sendo constituído de 3 tratamentos (espaçamentos de distribuição da isca) com 5 repetições compostas de 10 ninhos cada. Cada parcela foi constituída de faixas com 8 linhas de plantio e $24 \mathrm{~m}$ de comprimento.

Para a avaliação da eficiência de controle de formigas, foram selecionados em cada parcela 10 ninhos de até $1 \mathrm{~m}^{2}$ de área de terra solta. Sendo 5 ninhos na faixa onde $o$ trator aplicou a isca formicida e 5 na faixa ao lado da linha de aplicação. Esses ninhos foram marcados e sua inatividade avaliada aos 60 e 210 dias após aplicação (DAA). Na última avaliação, foram escavados com enxadas para comprovar se estavam mortos, segundo metodologia citada por Phillips et al. (1976). Os dados de percentagem de formigueiros externamente inativos foram submetidos à análise de variância e comparados pelo Teste de Tukey, a 0,05 de probabilidade.

A percentagem de carregamento de isca formicida por ponto também foi avaliada, seguindo metodologia citada por Reis (2009). Trinta doses de isca formicida foram coletadas individualmente na saída do equipamento e colocadas sobre papéis brancos com, aproximadamente, $10 \times 10 \mathrm{~cm}$, no centro das entrelinhas de plantio do eucalipto, simulando a aplicação do equipamento. Quinze pontos foram distribuídos na borda e o restante a $15 \mathrm{~m}$ do centro do talhão. $\mathrm{O}$ carregamento foi avaliado após 24 e 48 horas da aplicação, verificando-se a quantidade de isca restante em cada papel. Os dados foram submetidos à análise de variância e ao teste $\mathrm{F}$, comparandose o carregamento na borda e no centro do talhão.

A capacidade operacional efetiva do conjunto mecanizado trator-implemento foi avaliada nos três espaçamentos de deposição da isca. Para isto, determinou-se o tempo necessário para se realizar a distribuição da isca em área conhecida de cada talhão, considerando manobras e tempos perdidos.

Para avaliar a qualidade do processo de distribuição da isca, sob ponto de vista estatístico, empregou-se a metodologia das cartas de controle. Em cada espaçamento de distribuição de isca, determinou-se a massa de 40 doses coletadas aleatoriamente em sacos de papel junto à saída da máquina, construindo, posteriormente, as cartas de controle. Essas cartas foram feitas a partir da média, do limite de controle inferior e do limite de controle superior, elaboradas no programa Minitab ${ }^{\circledR}$. Para estimar os limites dos gráficos de controle, foram usadas as equações $\mathrm{LS}=\mathrm{x}+3 \sigma$ e $\mathrm{LI}=\overline{\mathrm{x}}-3 \sigma$, em que: LS - limite de controle superior; $\mathrm{LI}$ - limite de controle inferior; $x$ - média geral da variável; $\sigma$ - desvio-padrão.

\section{RESULTADOS E DISCUSSÃO}

Na Tabela I, tem-se a percentagem de ninhos inativos aos 60 e 210 dias após aplicação de isca formicida. Verificou-se que não houve diferença entre os tratamentos aos 60 e 210 dias após aplicação. Esperava-se que quanto menor a distância entre pontos de aplicação de isca formicida, maior seria a mortalidade dos ninhos, mas este fato não ocorreu. Isso pode ser devido a grande quantidade de ninhos pequenos existente no talhão e ao carregamento da isca granulada por formigas não alvo. O espaçamento de $12 \mathrm{~m}$ entre linhas de aplicação apresentou mortalidade de 72,67\% e reduziu o número de passadas da máquina pela área e, desta forma, diminuiu o gasto energético em áreas de baixa infestação de ninhos de formigas cortadeiras. Em pesquisa realiza realizada na mesma região utilizando mesma dosagem sistematicamente, obteve mortalidade de $87,33 \%$ (Reis et al., 2009). Em trabalho realizado em no cerrado de Minas Gerais, apresentou eficiência 75\% (Zanetti et al., 2008b), já na região de Mata Atlântica de Mineira, utilizando o combate sistemático a granel na dose de $5 \mathrm{~g}$ a cada $6 \mathrm{~m}^{2}$ e microporta-iscas na dose de $10 \mathrm{~g}$ a cada $12 \mathrm{~m}^{2}$, obtiveram mortalidades para as espécies Atta spp., de 13,30\% e 6,70\% (Zanetti et al., 2003). Utilizando o controle localizado os resultados mortalidade foram de $90 \%$ por ZANUNCIO et al. (2002a) e $86,7 \%$ por ZANETTI et al. (2004).

O carregamento de iscas pelas formigas cortadeiras foi semelhante $(F ; p>0,05)$ independente do local de estabelecimento do formigueiro, na borda ou centro do talhão após 24 horas (61,59\% e 65,70\%) e 48 horas $(70,60 \%$ e $80,40 \%)$ após a aplicação da isca. Resultados semelhantes foram encontrados em 24 horas (59,89\% e $64,27 \%)$ e 48 horas $(68,60 \%$ e $75,33 \%)$ por REIS (2009). Os valores encontrados foram inferiores ao de transporte médio após 24 horas da aplicação localizada nos ninhos (85,93\%) encontrado por Zanetti et al. (2004). Isto mostra que a eficácia do controle pode estar relacionada à atratividade da isca e ao potencial de forrageamento das formigas cortadeiras (CAMARGO et al., 2003; LOPES et al., 2003; PODEROSO et al., 2009; RIBEIRO et al., 2009; URBAS et al., 2007). 
TABELA 1 Porcentagem de ninhos de formigas cortadeiras inativos aos 60 e 210 dias após a aplicação (DAA) de isca formicida granulada sistematicamente, em diferentes espaçamentos de distribuição. Paraopeba, Minas Gerais, 2010.

TABLE 1 Percentage of inactive nests of leaf-cutting ants at 60 and 210 days after systematically application (DAA) of granular ant bait at different spacing distribution. Paraopeba, Minas Gerais, 2010.

\begin{tabular}{ccc}
\hline \multirow{2}{*}{$\begin{array}{c}\text { Espaçamento de } \\
\text { aplicação da isca }\end{array}$} & \multicolumn{2}{c}{$\%$ de ninhos inativos* } \\
\cline { 2 - 3 } 60 DAA & 210 DAA \\
\hline $9 \times 2,5 \mathrm{~m}$ & $35,12^{\mathrm{a}}$ & $73,81^{\mathrm{a}}$ \\
$12 \times 2,5 \mathrm{~m}$ & $57,50^{\mathrm{a}}$ & $87,50^{\mathrm{a}}$ \\
\hline
\end{tabular}

* Médias seguidas de mesma letra, nas colunas, não diferem significativamente entre si pelo teste de Tukey, a 0,05 de probabilidade.

A maior parte dos ninhos de formigas cortadeiras concentra-se nas bordas dos talhões (ZANUNCIO et al., 2002; RAMOS et al., 2008), sendo que sistema de aplicação sistemática distribui a isca na mesma quantidade, quer seja, na borda ou no centro dos talhões, não influenciando o carregamento pelas formigas.

$O$ rendimento operacional do equipamento de aplicação das iscas granuladas foi de 3,3।; 3,78 e 8,42 ha. $h^{-1}$ para os espaçamentos de $6 \times 2,5 \mathrm{~m}, 9 \times 2,5 \mathrm{~m}$ e $12 \times 2,5 \mathrm{~m}$, respectivamente. Em pesquisa realizada por Reis et al. (2009), o rendimento de um conjunto mecanizado foi de $2,28 \mathrm{ha} \cdot \mathrm{h}^{-1}$, correspondendo a 19,38 ha. $\mathrm{dia}^{-1}$, enquanto 0 controle manual atingiu valores próximos a 5 ha.dia ${ }^{-1}$ em áreas de Cerrado e $3 \mathrm{ha}^{-\mathrm{dia}^{-1}}$ em áreas de Mata Atlântica.

$\mathrm{Na}$ Figura I, têm-se as cartas de controle do processo de distribuição da isca formicida. Verificou-se que nos dois espaçamentos menores, não ocorreram pontos fora dos limites superior e inferior, indicando que o processo está sob controle estatístico. No maior espaçamento $(12 \times 2,5 \mathrm{~m})$, houve um único ponto abaixo do limite inferior, provalmente devido ao próprio sistema de distribuição da máquina e ondulações do relevo.

A análise das cartas de controle mostra que não ocorreu grande variabilidade entre os 40 pontos amostrais. A aplicação de agroquímicos com doses reduzidas implica a necessidade de maior cuidado com o processo de distribuição. Destaca-se que, nesta avaliação das cartas de controle, não está sendo avaliada a amplitude relativa em si, mas sua variabilidade entre os pontos amostrais.



(a)



(b)

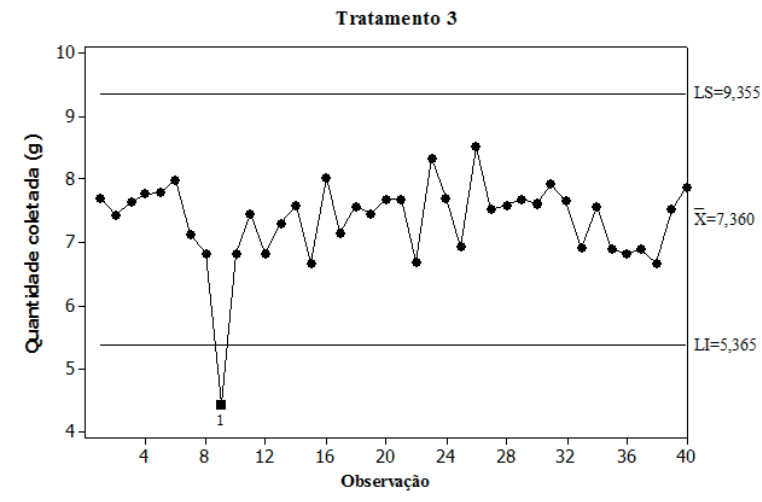

(c)

FIGURA 1 Cartas de controle do processo de distribuição da isca formicida nos três espaçamentos avaliados: a) $6 \times 2,5 \mathrm{~m}$; b) $9 \times$ $2,5 \mathrm{~m} \mathrm{e} \mathrm{c)} 12 \times 2,5 \mathrm{~m}$.

FIGURE 1 Control charts of the distribution process of the ant baits in three spacings evaluated: a) $6 \times 2.5 \mathrm{~m}$; b) $9 \times 2.5 \mathrm{~m}$ and c) $12 \times 2.5 \mathrm{~m}$.

Não foram encontrados na literatura trabalhos empregando cartas de controle para avaliação da qualidade da distribuição de isca formicida. Em outras áreas, o uso do controle estatístico de processos em operações agrícolas tem mostrado que resultados fora dos padrões pré-estabelecidos 
são freqüentemente obtidos (REIS et al., 20I0; SILVA et al., 2007), diferindo-se dos resultados deste trabalho.

\section{CONCLUSÕES}

Os diferentes espaçamentos não influenciaram a eficácia de controle de formigas cortadeiras. O maior espaçamento empregado ( $12 \times 2,5 \mathrm{~m}$ ) proporcionou maior capacidade operacional $8,42 \mathrm{ha} \cdot \mathrm{h}^{-1}$. A aplicação sistemática mecanizada proporcionou boa qualidade de distribuição, sob ponto de vista do controle estatístico de processo, constituindo alternativa viável à aplicação manual.

\section{AGRADECIMENTOS}

À Fundação de Amparo à Pesquisa do Estado de Minas Gerais (FAPEMIG), pela concessão da bolsa de pós-doutorado ao primeiro autor, à V\&M Florestal, pelo auxílio financeiro e apoio logístico nas operações de campo e à Unibrás Agroquímica Ltda., pelo empréstimo do aplicador de isca.

\section{REFERÊNCIAS BIBLIOGRÁFICAS}

ANDRADE, A. P. P.; FORTI, L. C.; MOREIRA, A. A.; BOARETTO, M. A. C.; RAMOS, V. M.; MATOS, C. A. O. Behavior of Atta sexdens rubropilosa (Hymenoptera: Formicidae) workers during the preparation of the leaf substrate for symbiont fungus culture. Sociobiology, Chicago, v. 40, n. 2, p. 293-306, 2002.

BONILLA, J. A. Qualidade total na agricultura: fundamentos e aplicações. 2.ed. Belo Horizonte: Centro de Estudos de Qualidade Total na Agricultura, 1994. 344 p.

CAMARGO, R. S.; FORTI, L. C.; MATOS C. A. O.; LOPES, J. F.; ANDRADE, A. P. P.; RAMOS V. M. Post-selection and return of foraged material by Acromyrmex subterraneus brunneus (Hymenoptera: Formicidae). Sociobiology, Chicago, v. 42, n. I, p. 93-102, 2003.

LOPES, J. F. S.; FORTI, L. C.; BOARETTO, M. A.; CAMARGO, R. S.; ANDRADE, A.P.; RAMOS, V. M.; NAGAMOTO, N. S. Devolution rates of grass by Atta capiguara (Hymenoptera, Formicidae) in Weld conditions. Pasturas tropicales, Cali, v. 25, n. I, 42-45, 2003.

MINGOTI, S. A.; FIDELIS, M. T. Aplicando a geoestatística no controle estatístico de processo. Revista Produto \& Produção, Porto Alegre, v. 5, n. 2, p.55-70, 200 I.

MONTGOMERY, D. C. Introduction to statistical quality control. New York: John Wiley, I99I. 677 p.
NAGAMOTO, N. S.; FORTI, L. C.; RAETANO, C. G. Evaluation of the adequacy of dixubenzuron and dechlorane in toxic baits for leaf-cutting ants (Hymenoptera: Formicidae) based on formicidal activity. Journal of Pest Science, Heidelberg, v. 80, n. I, p. 9-13, 2007.

NAGAMOTO, N. S.; FORTI, L. C.; ANDRADE, A. P. P.; BOARETTO, M. A. C.; WILCKEN, C. F. Method for the evaluation of insecticidal activity over time in Atta sexdens rubropilosa workers (Hymenoptera: Formicidae). Sociobiology, Chicago, v. 44, n. 2, p. 4I3-432, 2004.

NICHOLS-ORIANS, C. M. Environmentally induced differences in plant traits: consequences for susceptibility to a leafcutter ant. Ecology, Ithaca, v. 72, n.5, p. 1609-1623, I99I.

PHILLIPS, F. T.; ETHERIDGE, P.; SCOTT, G. C. Formulation and field evaluation of experimental baits for the control of leaf-cutting ants (Hymenoptera: Formicidae) in Brazil. Bulletin of Entomological Research, London, v. 66, p. 579-585, 1976.

PODEROSO, J. C. M.; RIBEIRO, G. T.; GONÇALVES, G. B.; MENDONÇA, P. D.; POLANCZYK, R. A.; ZANETTI, R.; SERRÃO J. E.; ZANUNCIO, J. C. Nest and foraging characteristics of Acromyrmex landoltibalzani (Hymenoptera: Formicidae) in Northeast Brazil. Sociobiology, Chico, v. 54, n.2, p. 36I-37I, 2009.

RAMOS, V. M.; FORTI, L. C.; ANDRADE, A. P. P.; NORONHA, N. C.; CAMARGO, R. S. Density and spatial distribution of Atta sexdens rubropilosa and Atta laevigata colonies (Hym., Formicidae) in Eucalyptus spp. forests. Sociobiology, Chicago, v. 5I, n. 3, p. 775-782, June 2008.

REIS, E. F.; QUEIROZ, D. M.; CUNHA, J. P. A. R.; ALVES, S. M. F. A. Qualidade da aplicação aérea líquida com uma aeronave agrícola experimental na cultura da soja (Glycine max L.). Engenharia Agrícola, Jaboticabal, v.30, n.5, p.958-966, set./out. 2010.

REIS, M. A. Avaliação e aperfeiçoamento de programas de manejo de formigas cortadeiras (Hymenoptera: Formicidae) em eucaliptais. 2009. 66 p. Tese (Doutorado Entomologia) - Universidade Federal de Lavras, Lavras, MG.

REIS, M. A.; ZANETTI, R.; SCOLFORO, J. R. S.; FERREIRA, M. Z.; ZANUNCIO J. C. 2008. Sampling of leaf-cutting ant nests (Hymenoptera: Formicidae) in eucalyptus plantations using quadrant and Prodan methods. Sociobiology, Chicago, v. 5I, n. I, p. 2I-29, 2008.

REIS, M. A.; ZANETTI, R.; FERNANDES, B. V. Controle sistemático mecanizado de formigas cortadeiras com isca granulada em eucaliptais em fase de manutenção. In: XIX Simpósio de Mirmecologia, 19, 2009, Ouro Preto. Anais... Ouro Preto, 2009.

RIBEIRO, P. L.; HELENE, A. F.; XAVIER, G.; NAVAS, C.; RIBEIRO. F. L. Ants can learn to forage on one-way trails. Plos One, Cambridige, v. 4, n. 4, p. I-7, 2009. 
SILVA, R. P.; SOUZA, F. G.; CORTEZ, J. W.; FURLANI, C. E. A.; VIGNA, G. P. Variabilidade espacial e controle estatístico do processo de perdas na colheita mecanizada do algodoeiro. Engenharia Agrícola, Jaboticabal, v.27, n.3, p.742-752, set./dez. 2007.

URBAS, P.; ARAÚJO Jr., M.V.; LEAL, I. R.; WIRTH, R. Cutting more from cut forests: edge effects on foraging and herbivory of leaf-cutting ants in Brazil. Biotropica, Malden, v. 39, n. 4, p.489-495, 2007.

UKAN, D. Avaliação qualitativa e quantitativa de microporta iscas para o controle de formigas cortadeiras, em plantios de Eucalyptus urograndis submetidos a diferentes cronogramas silviculturais. 2008. 76 p. Dissertação (Mestrado em Ciências Florestais) Universidade Federal do Paraná, Curitiba.

ZANETTI, R.; ZANUNCIO, J. C.; VILELA, E. F.; LEITE, H. G., JAFFÉ, K.; OLIVEIRA A. C. Level of economic damage for leaf-cutting ants in Eucalyptus plantations in Brazil. Sociobiology, Chicago, v. 42, n. 2, p. 433-442, 2003.
ZANETTI, R.; ZANUNCIO, J. C.; MAYHÉ-NUNES, A. J.; MEDEIROS, A. G. B.; SOUZA-SILVA, A. Combate sistemático de formigas-cortadeiras com iscas granuladas, em eucaliptais com cultivo mínimo. Revista Árvore, Viçosa, MG, v. 27, n. 3, p. 387-392, maio/jun. 2003.

ZANETTI, R.; DIAS, N.; REIS, M. A.; SOUZA-SILVA, A.; MOURA, M. A. Eficiência de iscas granuladas (sulfluramida 0,3\%) no controle de Atta sexdens rubropilosa Forel, 1908 (Hymenoptera: Formicidae). Ciência e Agrotecnologia, Lavras, v. 28, n. 4, p. 878-882, jul./ago. 2004.

ZANETTI, R.; ZANUNCIO, J. C.; SOUZA-SILVA, A.; MENDONÇA, L. A.; MATTOS, J. O. S.; RIZENTAL, M. S. Eficiência de produtos termonebulígenos no controle de Atta laevigata (Hymenoptera: Formicidae) em plantio de eucalipto. Ciência e Agrotecnologia, Lavras, v. 32, n. 4, p. I313-1316, jul./ago. 2008

ZANUNCIO, J. C.; LOPES, E. T.; ZANETTI, R.; PRATISSOLI, D.; COUTO, L. Spatial distribution of nests of the leafcutting ant Atta sexdens rubropilosa (Hymenoptera: Formicidae) in plantations of Eucalyptus urophylla in Brazil. Sociobiology, Chicago, v. 39, n. 2, p. 23I-242, Feb. 2002. 